\title{
A case of pediatric psoriasis achieving remission after allogenic bone marrow transplantation
}

\author{
Dilara Güler $^{1 \oplus}$, Gülhan Gürel ${ }^{1 \oplus}$, Gülsüm Şeyma Yalçın², İbrahim Eker ${ }^{3 \oplus}$, \\ İrem Nur Durusu ${ }^{1 \oplus}$, Çiğdem Özdemir ${ }^{2 \oplus}$, Özge Vural ${ }^{4 \oplus}$ \\ Departments of ${ }^{1}$ Dermatology, ${ }^{2}$ Pathology and ${ }^{3}$ Pediatric Hematology, Afyonkarahisar Health Sciences University Afyonkarahisar; \\ ${ }^{4}$ Department of Pediatric Hematology, Gazi University Faculty of Medicine, Ankara, Turkey.
}

\begin{abstract}
Background. Psoriasis is an inflammatory skin disease in which the cells and molecules of innate and adaptive immunity are involved in the pathogenesis. Aplastic anemia is a bone marrow deficiency syndrome that is characterized by an extreme reduction in the number of blood cells as a result of failure in hematopoiesis. Allogenic hematopoietic stem cell transplantation is a promising treatment for Aplastic Anemia and it is important to note that other comorbid diseases like psoriasis- since both have some common pathogenetic mechanisims- might achieve remission after treatment.
\end{abstract}

Case. We present a 12-years-old male patient who underwent bone marrow transplantation for aplastic anemia and his psoriasis vulgaris lesions completely regressed. The final follow-up visit on day 150 also revealed no sign of the pre-transplantation skin and scalp lesions.

Conclusions. This is the first case of pediatric psoriasis together with aplastic anemia that achieved complete remission of psoriasis after bone marrow transplantation. Our case report needs to be supported by prospective studies involving larger patient populations.

Key words: aplastic anemia, autoimmune, bone marrow transplantation, pediatric, psoriasis.

Psoriasis is an inflammatory disease that is mainly associated with dermatological manifestations, and affects approximately 2-3\% of the general population. ${ }^{1}$ The prominent mechanisms in the pathogenesis of psoriasis are immune-mediated processes. Previous studies have demonstrated that the infiltration of the dermis and epidermis by CD4+ and CD8+ T cells and dendritic cells plays a key role in its pathogenesis, hence the cytokines released from these cells are involved in the immune processes. $^{2,3}$

\footnotetext{
凶 Dilara Güler

dsaritepe78@gmail.com
}

Received 10th December 2020, revised 31st January 2021, 17th February 2021, 13th April 2021, accepted 9th May 2021.

This case report was presented as oral presentation in the International Dermatology, Dermatopathology and Esthetics Academy 2020 Virtual Congress held on 19-22 November 2020.
Aplastic anemia is a bone marrow deficiency syndrome that is characterized by an extreme reduction in the number of blood cells as a result of failure in hematopoiesis. Immune-mediated mechanisms manifest in the destruction of hematopoietic cells by lymphocytes are involved in the occurrence of aplastic anemia. A vast majority of patients diagnosed with severe aplastic anemia are cured with allogenic stem cell transplantations from a histocompatible sibling. ${ }^{4,5}$

Patients undergoing allogeneic stem cell transplantations may also experience remission in several immune-mediated diseases that are comorbid with aplastic anemia. Previous studies have reported the remission of psoriasis following allogenic stem cell transplantation, most frequently in adult patients in which the transplant indication was malignancy. ${ }^{6-8} \mathrm{~A}$ further study in literature reports on a pediatric 
case with psoriasis improved remission after a transplantation for Ewing's sarcoma. ${ }^{9}$ Herein, we present a case of pediatric psoriasis that achieved complete remission upon bone marrow transplantation.

\section{Case Report}

A 12-years-old male patient who diagnosed with psoriasis four years previously was admitted to the pediatric hematology clinic with fever and fatigue. Physical examination revealed leukoplakia of the hard palate, diffuse scaly, erythematous plaques and petechial rash all over the body. There was nothing unusual in the patient's medical history, aside from psoriasis, nor any drug and/or toxic substance exposure that could cause pancytopenia. A complete blood count revealed severe pancytopenia. A bone marrow aspiration revealed cellularity of $<5 \%$, there were no megakaryocytes, while rare myelocytic and erythrocytic series were noted. The patient was thus diagnosed with aplastic anemia. While attempting to determine the etiology of the aplastic anemia, the patient was administered blood component transfusions, when he developed symptomatic anemia and thrombocytopenia, as well as supportive therapies involving antimicrobial treatments when he was febrile. While the patient was undergoing treatment in this regard, the patient was consulted to the department of Dermatology due to an increase in the psoriatic lesions. It was ascertained from the patient's medical history that he had presented to the Dermatology outpatient clinic many times before with nummular, scaly, erythematous plaque lesions on the trunk, left elbow and inguinal region for about $1.5-2$ years. The first histopathological examination had been performed 4 years ago and was reported as psoriasis vulgaris. The patient was treated with topical corticosteroids, topical calcipotriol and methotrexate at different times. He had only been receiving intermittent topical corticosteroid therapy for the previous six months.

Dermatological assessment revealed annular, scaly, erythematous plaques on the trunk and upper extremity. Scaly, mildly erythematousto-yellowish, greasy plaques were also noted around the eyes and scalp (Fig. 1abc). Upon the recent dermatological assessment a new punch biopsy was performed. The histopathological examination revealed parakeratotic foci on the surface, scale crust formations within parakeratoses and loss of granular cell layer immediately underneath the parakeratotic foci. There were granular cell layer of 2-3 lines in the non-parakeratotic areas. As well as irregular acanthosis, mild spongiosis, occasional lymphocyte exocytosis and focal suprapapillary thinning were seen. There were

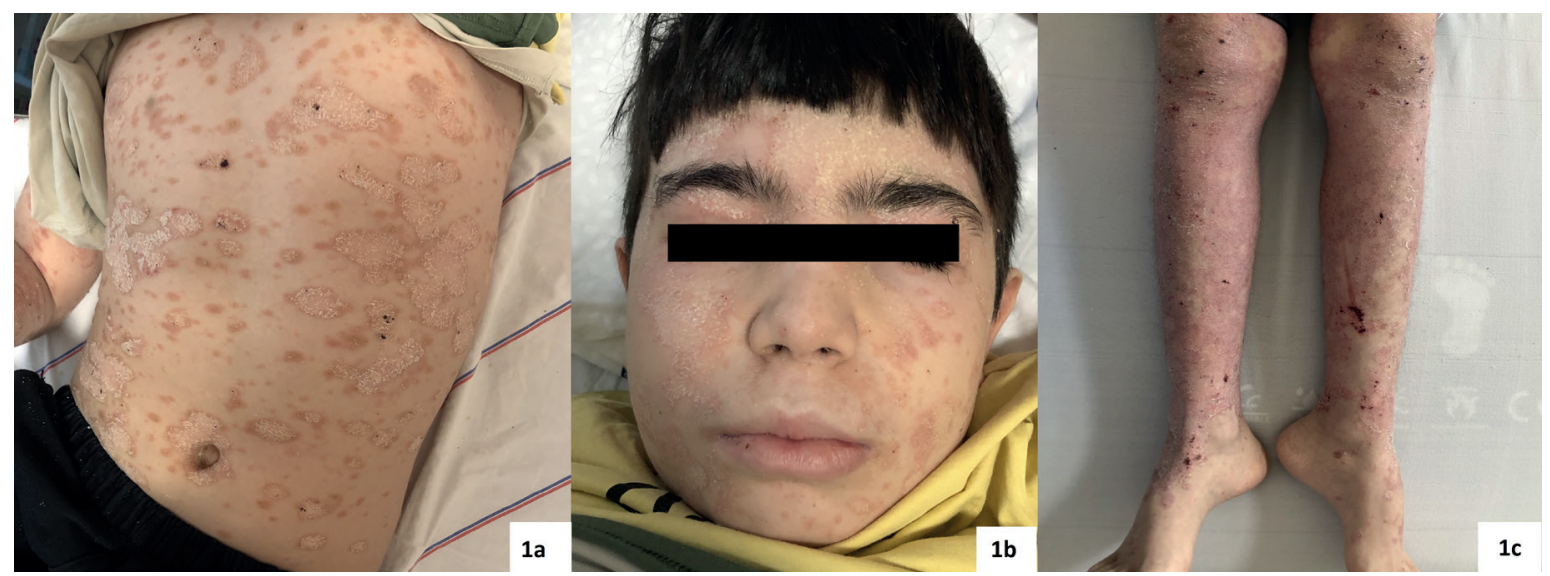

Fig. 1abc. Annular plaque on an erythematous base covered with squamae on the trunk and upper extremities, and squamous plaque lesions with erythema and a yellowish, greasy look around the eyes and on the scalp before treatment. 
tortuous, dilated and congested capillaries in some areas of papillary dermis. The superficial dermis featured with scattered melanophages and slight perivascular lymphocytic infiltrate around the superficial vascular plexus. Deeper sections indicated that the parakeratotic foci had a tendency to be located at the follicular ostia (Fig. 2abc).

Considering the clinical and histopathological findings, the patient was diagnosed with sebopsoriasis, and treatment was initiated with systemic itraconazole, topical corticosteroids and ketoconazole. At the same time period examinations were made to investigate the etiology and to classify the aplastic anemia and resulted with the diagnosis of a very severe acquired aplastic anemia. The bone marrow transplant had to be delayed due to the COVID-19 pandemic, and the patient required more transfusions and antimicrobial supportive therapies as time went by. Seven months after the aplastic anemia diagnosis, the patient underwent a bone marrow transplantation from his fully-matched brother without any psoriasis diagnosis. A conditioning regimen was administered using cyclophosphamide (total dose $200 \mathrm{mg} / \mathrm{kg}$ intravenously) over 4 days and ATG Fresenius (total dose $35 \mathrm{mg} /$ $\mathrm{kg}$ ) intravenously over 4 days. Umbilical cord-derived mesenchymal stem cells (MSC) were applied for graph versus host disease (GVHD) prophylaxis, and cyclosporine A and methotrexate were used. Dermatological assessment on day 7 following the allogeneic stem cell transplantation, revealed complete remission of the cutaneous lesions of the patient (Fig. 3abc). He was discharged on day 43 with follow-up by the Pediatric Hematology department on an outpatient basis. The patient experienced no complications during follow-up in the outpatient setting, and the final followup visit on day 150 also revealed no sign of the pre-transplantation skin and scalp lesions. We obtained consent from his family to publish this report and to include his photograph.

\section{Discussion}

Psoriasis is an inflammatory skin disease in which the cells and molecules of innate and adaptive immunity are involved in the pathogenesis. ${ }^{10}$ While it may occur in any period of life, psoriasis has two peak onset periods at the age of 20-30 years and at the age of 50-60 years. Symptoms are reported to start under the age of 20 years in $35-50 \%$ of patients. ${ }^{11}$ Primary cells involved in the pathogenesis of the disease are dendritic cells, Th1, Th17 and Th22 lymphocytes. IL-12 and IL-23 released from the inflammatory myeloid dendritic cells induce the release of IL-17, IFN- $\gamma$, TNF- $\alpha$ and

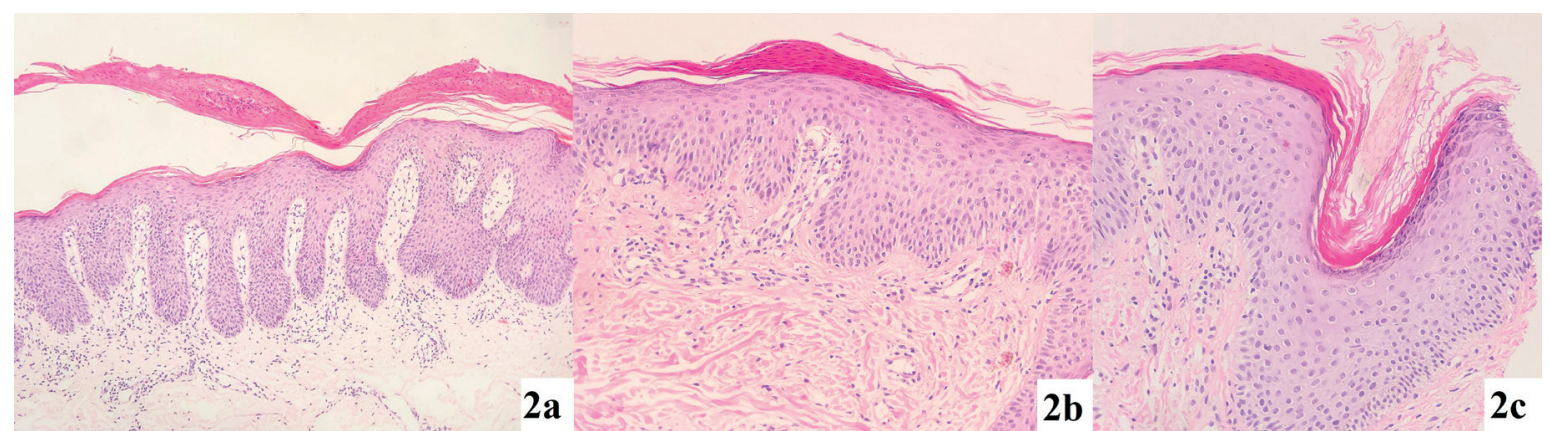

Fig. 2abc. 2a: Patient's first punch biopsy revealed features consistent with Psoriasis (confluent parakeratosis, neutrophilic parakeratosis, loss of granular cell layer, regular acanthosis, elongated rete ridges, suprapapillary thinning, dilated capillaries)(Hematoxylin and eosin, x100) 2b: Patient's recent punch biopsy showed parakeratotic focus, loss of granular cells underneath the parakeratosis, minimal spongiosis, tortuous and dilated capillaries in the dermal papilla and minimal perivascular lymphocytic infiltrate (Hematoxylin and eosin, x200) 2c: The parakeratotic focus was located at the follicular ostium in deeper sections of the patient's recent biopsy (Hematoxylin and eosin, x200). 

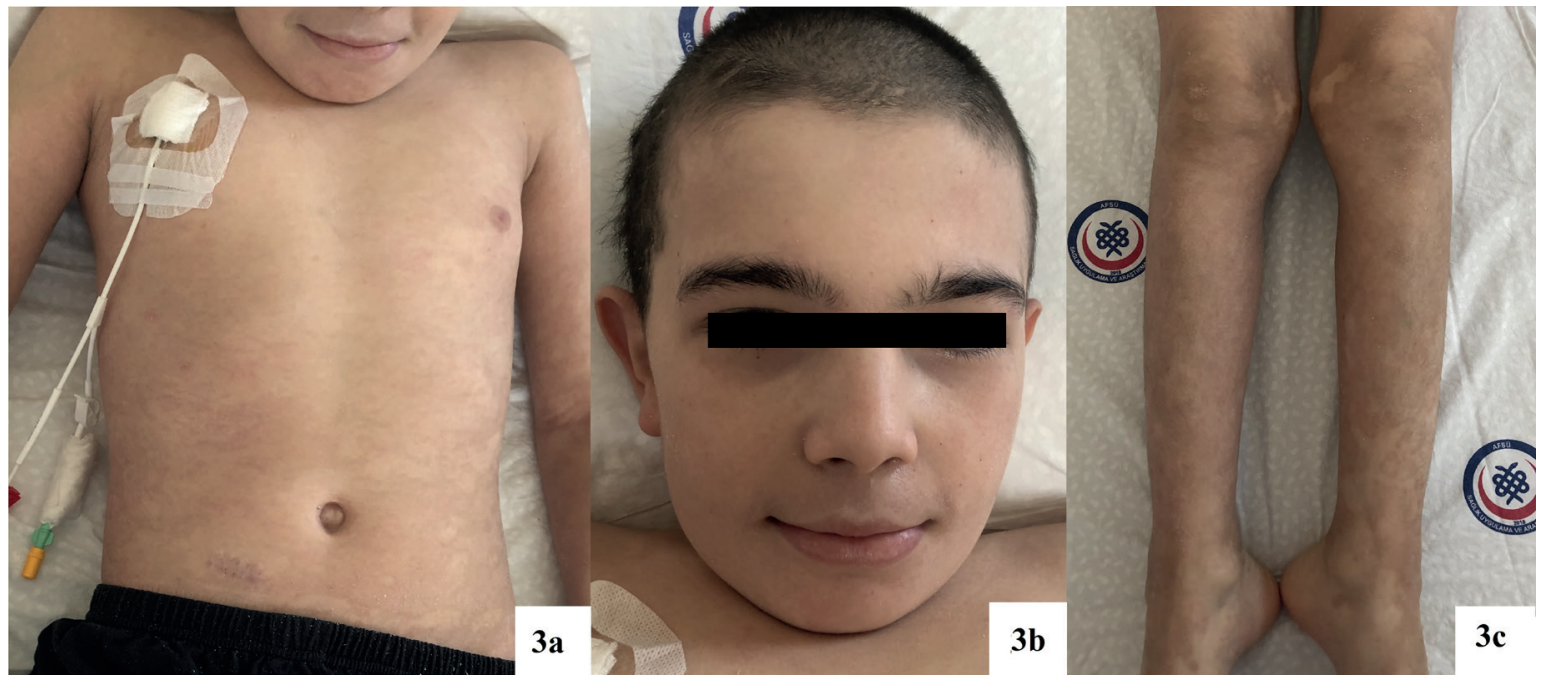

Fig. 3abc. Complete remission in psoriasis vulgaris at visit on the 7th day after bone marrow transplantation.

IL-22 -which are known as psoriatic cytokinesfrom Th1, Th17 and Th22. ${ }^{10}$

Aplastic anemia is a disease characterized by pancytopenia and hypoplastic bone marrow. ${ }^{12}$ Previous studies have found that abnormally activated CD8+ T lymphocytes, dendritic myeloid cells, Th1 cells and some small molecules released from them such as IFN- $\gamma$, TNF- $\alpha$ and IL- 2 play the main role in pathogenesis of the disease. ${ }^{13}$ The etiology of aplastic anemia includes viral infections, environmental toxins, inherited and acquired mutations. The curative treatment of the disease is bone marrow transplantation. ${ }^{14}$

Complete remission of psoriasis after bone marrow transplantation for aplastic anemia has not been reported in a child. In the literature, there are some case reports that have shown remission in psoriasis after bone marrow transplantation. ${ }^{6-8}$ A 40-year-old male patient reported by Kishimoto et al. ${ }^{6}$ achieved a complete remission in palmoplantar pustular psoriasis following bone marrow transplantation for the treatment of Acute Myelocytic Leukemia (AML). Another case reported by Yokota et al. ${ }^{7}$ 36-year-old male patient with psoriasis vulgaris who developed drug-related aplastic anemia and achieved complete remission in psoriatic plaque and nail symptoms, despite not receiving any antipsoriatic treatment after the bone marrow transplantation. We believe that the alteration of the host's immune system cells may have been involved in the remission of the psoriasis.

Occurrence of GVHD has significant correlation with the number of platelet transfusions and the number of red blood cell (RBC) transfusions in allogeneic hematopoietic stem cell transplantation (HSCT) from HLA-matched sibling donors for severe aplastic anemia. Our patient had a high transfusion burden of platelet and $\mathrm{RBC}$ before transplant and because of this he had a significant risk of GVHD. ${ }^{10}$ On the other hand, there are some studies reporting increased T cell activation in patients with aplastic anemia. Some clinical conditions which have been seen in aplastic anemia may be due to mesenchymal disorders. MSC infusion may be an effective treatment modality in aplastic anemia. MSC infusion inhibits T-cell-mediated hematopoietic stem cell (HSC) destruction and aids in the formation of the environment that contributes to hematopoiesis. There are some studies using MSC infusion alone or in combination with HSCT in the treatment of aplastic anemia. ${ }^{11}$ Autoreactive T-lymphocytes are also thought to contribute to psoriasis through the ineffective control of proinflammatory cells. ${ }^{12}$ For all these reasons, in our patient with both psoriasis, 
aplastic anemia and heavy transfusion load, MSC was given in the aim to reduce the risk of GVHD, increase the likelihood of engraftment and we hoped it would be effective in treating psoriasis, despite the transplantation from HLA full matched sibling.

The main emphasis of our case report is the effect of HSCT on psoriasis. HSCT indication for this case was very severe acquired aplastic anemia. But after the transplantation, the patient's psoriasis also went into remission completely. Basically, the elimination of the autoreactive clone in the bone marrow with HSCT, that causes psoriasis, may explain the remission of the autoimmune disease after transplantation. Additionally, the high dose of immunosuppressive treatment given during the conditioning regimen may be effective in the remission of the disease. This explains the remission of psoriasis as early as 7 days after HSCT when the effects of the transplanted clones are not observed yet. Another important point is the successful engraftment in the patient, although the amount of CD34 positive stem cells that can be given to the patient is very low. MSC therapy applied to the patient may also have been effective in providing this, by modulating the immune responses and maintaining an environment supportive of hematopoiesis. ${ }^{13}$

In a study, the status of psoriasis after allogenic HSC transplantation was evaluated in patients with diagnosis of psoriasis with hematological malignancies. Ciurea et al. ${ }^{14}$ demonstrated that allogeneic transplantation is effective in the treatment of disease in patients with psoriatic skin lesions and arthritis. They saw a rapid recovery after transplantation and the lesions did not recurred in the seven-year followup. ${ }^{14}$ Kaffenberger et al. ${ }^{15}$ also suggested that psoriasis is likely to remit after allogeneic HSCT, but it is likely to recur after autologous HSCT, in their report of 19 cases with psoriasis and had undergone allogenic or autologous HSCT. MSCs were not used in these transplants also.
Neither Ciurea nor Kaffenberger give information in their reports about the chimerism of the patients. But Chakrabarti et al. ${ }^{16}$ reported a patient with severe psoriasis achieved remission after nonmyeloablative allogeneic HSCT due to non-Hodgkin's lymphoma. MSCs were not used in this transplant also. They concluded that if long-term remission is achieved in autoimmune diseases after allogeneic HSCT with lowintensity conditioning regimens, allogeneic transplantation may become an option for the treatment of some autoimmune diseases. All of these findings suggested that in our patient the allogenic HSCT may be the prominent cause of psoriasis remission rather than MSC.

\section{Author contribution}

The authors confirm contribution to the paper as follows: study conception and design: DG, GG, IE; data collection: DG,GSY, İE, CO, $\mathrm{OV}$; analysis and interpretation of results: DG,GG,GSY, IE; draft manuscript preparation: DG, GG, GSY, İE, İND. All authors reviewed the results and approved the final version of the manuscript.

\section{Conflict of interest}

The authors declares that there is no conflict of interest regarding the publication of this paper.

\section{REFERENCES}

1. Henry A, Kyle S, Chisholm A, Griffiths CEM, Bundy C. A cross-sectional survey of the nature and correlates of sleep disturbance in people with psoriasis. Brit J Dermatol 2017; 177: 1052-1059. https://doi.org/10.1111/bjd.15469

2. Boehncke WH. Etiology and pathogenesis of psoriasis. Rheum Dis Clin North Am 2015; 41: 665675. https://doi.org/10.1016/j.rdc.2015.07.013

3. Li X, Li J, Wang L, et al. Transmission of psoriasis by allogeneic bone marrow transplantation and blood transfusion. Blood Cancer J 2015; 5: e288. https://doi. org/10.1038/bcj.2015.15

4. Young NS. Acquired aplastic anemia. Ann Intern Med 2002; 136: 534-546. https://doi.org/10.7326/00034819-136-7-200204020-00011 
5. Young NS, Calado RT, Scheinberg P. Current concepts in the pathophysiology and treatment of aplastic anemia. Blood 2006; 108: 2509-2519. https:// doi.org/10.1182/blood-2006-03-010777

6. Kishimoto $\mathrm{Y}$, Yamamoto $\mathrm{Y}$, Ito $\mathrm{T}$, et al. Transfer of autoimmune thyroiditis and resolution of palmoplantar pustular psoriasis following allogeneic bone marrow transplantation. Bone Marrow Transplant 1997; 19: 1041-1043. https://doi. org/10.1038/sj.bmt.1700789

7. Yokota A, Hukazawa M, Nakaseko C, et al. Resolution of psoriasis vulgaris following allogeneic bone marrow transplantation for aplastic anemia. Rinsho Ketsueki 1996; 37: 35-39.

8. Eedy DJ, Burrows D, Bridges JM, Jones FG. Clearance of severe psoriasis after allogenic bone marrow transplantation. BMJ 1990; 300: 908. https:// doi.org/10.1136/bmj.300.6729.908

9. Held K, Rahmetulla R, Loew TW, Radhi MA. Complete resolution of guttate psoriasis following autologous SCT for Ewing's sarcoma in a pediatric patient. Bone Marrow Transplant 2012; 47: 15851586. https://doi.org/10.1038/bmt.2012.68

10. Chen X, Wei JL, Huang Y, et al. Outcome of allogeneic hematopoietic stem cell transplantation from HLAmatched sibling donor for 41 cases of severe aplastic anemia. Zhonghua Xue Ye Xue Za Zhi 2012; 33: 610614.
11. Broglie L, Margolis D, Medin JA. Yin and Yang of mesenchymal stem cells and aplastic anemia. World J Stem Cells 2017; 9: 219-226. https://doi.org/10.4252/ wjsc.v9.i12.219

12. Boehncke WH, Brembilla NC. Autoreactive T-lymphocytes in inflammatory skin diseases. Front Immunol 2019; 10: 1198. https://doi.org/10.3389/ fimmu.2019.01198

13. Chao YH, Tsai C, Peng CT, et al. Cotransplantation of umbilical cord MSCs to enhance engraftment of hematopoietic stem cells in patients with severe aplastic anemia. Bone Marrow Transplant 2011; 46: 1391-1392. https://doi.org/10.1038/bmt.2010.305

14. Ciurea SO, Hansrivijit P, Ciurea AM, et al. Curative potential of hematopoietic stem cell transplantation for advanced psoriasis. Am J Hematol 2019; 94: E176-E180. https://doi.org/10.1002/ajh.25470

15. Kaffenberger BH, Wong HK, Jarjour W, Andritsos LA. Remission of psoriasis after allogeneic, but not autologous, hematopoietic stem-cell transplantation. J Am Acad Dermatol 2013; 68: 489-492. https://doi. org/10.1016/j.jaad.2012.08.021

16. Chakrabarti S, Handa SK, Bryon RJ, Griffiths MJ, Milligan DW. Will mixed chimerism cure autoimmune diseases after a nonmyeloablative stem cell transplant? Transplantation 2001; 72: 340-342. https://doi.org/10.1097/00007890-200107270-00032 